\title{
Quantitative Evaluation of Copper Nano Cluster Combination Process by Multi Vacancy Lattice Monte Carlo Simulation
}

\author{
Tadao FUKUTA and Koichi OZAKI* \\ Department of System Engineering, Okayama Prefectural University, Soja 719-1197, Japan \\ Fax: 81-(0)866-94-2199, e-mail: fukuta@cse.oka-pu.ac.jp \\ * Department of System Engineering, Okayama Prefectural University, Soja 719-1197, Japan \\ Fax: 81-(0)866-94-2199, e-mail: ozaki@cse.oka-pu.ac.jp
}

\begin{abstract}
In this study, we focus on nanoscale copper-rich precipitates and attempt to elucidate their effect on the embrittlement of reactor pressure vessel steels. Our final goal is to evaluate such embrittlement from a microscopic viewpoint on the basis of an atomistic simulation. We develop a multi vacancy lattice Monte Carlo (MVLMC) method that can analyze a model having more than one vacancy. We show that the process of copper clustering consists of two phases-the formation phase and the combination phase. Further, we carry out a detailed analysis of the combination process of a cluster using MVLMC and evaluate the formation mechanism of a cluster using the distance between clusters. We find that when a nanocluster smaller than $1 \mathrm{~nm}$ and a cluster of approximately $2 \mathrm{~nm}$ coalesce, minute nanoclusters separate from the former cluster and are absorbed by the latter cluster.
\end{abstract}

Key words: Lattice Monte Carlo, Copper Cluster, Atomic Simulation

\section{INTRODUCTION}

The life of a nuclear reactor pressure vessel is one of the factors governing the life of a nuclear power plant. The ductile-brittle transition temperature (DBTT) is related to the irradiation hardening characteristic of yield strength and tensile strength. In a tensile test of pressure vessel steel damaged by neutron irradiation, the increase in tensile strength is smaller than that in yield strength. As a result, the strain-hardening coefficient decreases, and elongation after fracture also decreases. Therefore, the pressure vessel steel damaged by neutron irradiation may become brittle at room temperature because the DBTT is influenced by these phenomena.

In recent studies, direct observation of a material surface on the nanoscale has been successfully carried out as a result of the development of high-efficiency microscopes such as a transmission electron microscope (TEM). Such observations have elucidated that $\mathrm{Cu}$ atoms, which are contained in the pressure vessel steel, precipitate as clusters with progressing neutron damage [1]. Further, there is a strong possibility that the interaction between precipitates and dislocations is one of the causes of hardening. It is usually thought that $0.4 \mathrm{wt} \% \mathrm{Cu}$ at $973 \mathrm{~K}$ and $1.8 \mathrm{wt} \% \mathrm{Cu}$ at $1123 \mathrm{~K}$ are in a solid solution state in $\alpha-\mathrm{Fe}$ [2]. It is considered that $\mathrm{Cu}$ in the pressure vessel steel gradually precipitates through vacancies formed by neutron irradiation.

Among the several experimental studies conducted on nanoscale $\mathrm{Cu}$ precipitates, the study by Hardouin et al. [3] has confirmed, by means of TEM observation, that nanoscale $\mathrm{Cu}$ precipitates are formed in $\mathrm{Fe}-1.5 \mathrm{wt} \% \mathrm{Cu}$ alloy under electron irradiation of $2.5 \mathrm{MeV}\left(1.4 \times 10^{-3}\right.$ $\left.\mathrm{dpa}, 2.0 \times 10^{-9} \mathrm{dpa} \cdot \mathrm{s}^{-1}\right)$ at $568 \mathrm{~K}$. Further, Nagai et al. [4] performed a coincidence Doppler broadening (CDB) measurement of positron annihilation and elucidated that a positron is closed in $\mathrm{Cu}$ nanoscale precipitates and that these precipitates are formed under neutron irradiation and by thermal aging. In contrast, among the several numerical studies conducted on nanoscale $\mathrm{Cu}$ precipitates, the study by Soisson et al. [5] elucidated the formation process of $\mathrm{Cu}$ precipitates by a Monte Carlo (MC) simulation and demonstrated the validity of this simulation using a residence time algorithm. Schmauder and Binkele [6] enhanced the MC simulation of the formation process of $\mathrm{Cu}$ precipitates. Further, they applied the Russel-Brown theory to their simulation results in order to calculate the increase in the yield stress in the thermal aging stage, and they concluded that yield stress changes with a change in annealing time. They also calculated the motion of an edge dislocation in the $\mathrm{Fe}-\mathrm{Cu}$ matrix using the molecular dynamics (MD) method [7].

As mentioned above, experimental and numerical studies on nanoscale $\mathrm{Cu}$ precipitates have attempted to explain their embrittlement mechanism. Further, it is known that several types of vacancies are related to $\mathrm{Cu}$ precipitation. However, no researchers have attempted to introduce more than two vacancies into an analysis system using lattice Monte Carlo (LMC). In this study, we develop a new technique that is based on LMC with the same vacancy jump model as those of F. Soisson et al. and S. Schmauder et al. We improve this technique in order to use it for analysis of a model having more than two vacancies and call it the multi vacancy lattice Monte Carlo (MVLMC) method. Further, we elucidate the process of nanoscale $\mathrm{Cu}$ precipitation using the developed method, where the activation energy is calculated from the first neighboring interaction model.

\section{MVLMC SIMULATION METHOD}

2.1 Mechanism of Vacancy Jump

In the present study, we assume that interatomic 
energy acts on only the nearest-neighbor atoms. The total potential energy is estimated under the assumption that the cohesive energy of the alloy is a sum of pair interactions, $e_{i j}(i, j=F e, C u$, or $\mathrm{V})$. In a stable configuration, the total potential energy in the matrix is obtained by the following equation:

$$
\begin{aligned}
E & =N_{\mathrm{FeFe}} \varepsilon_{\mathrm{FeFe}}+N_{\mathrm{CuCu}} \varepsilon_{\mathrm{CuCu}}+N_{\mathrm{FeCue}} \varepsilon_{\mathrm{FeC} u} \\
& +N_{\mathrm{FeV}} \varepsilon_{\mathrm{FeV}}+N_{\mathrm{Cu} V} \varepsilon_{\mathrm{Cu} V}+N_{V V} \varepsilon_{V V}
\end{aligned}
$$

where $\mathrm{N}_{\mathrm{ij}}$ is the number of nearest-neighbor i-j pairs; "ghost" interaction energies between vacancies and others $\left(\varepsilon_{\mathrm{FeV}}, \varepsilon_{\mathrm{CuV}}\right.$, and $\left.\varepsilon_{\mathrm{VV}}\right)$ have been introduced.

Generally speaking, the total potential energy of a system must progress through activated state from one stable state to another stable state. Only a cell containing an atom $\mathrm{i}$ and a vacancy $\mathrm{V}$ is affected at a vacancy jump. The activation energy $\Delta \mathrm{E}_{\mathrm{iv}}$ is the increase in energy with the motion of atom $i$, the nearest-neighbor atom of the vacancy $\mathrm{V}$, from its stable site to the saddle point position, which is defined as follows:

$$
\Delta E_{i V}=E_{s}^{(i)}-\sum_{j \in n n(i)} \varepsilon_{i j}-\sum_{\substack{j \in n n(V) \\ j \neq i}} \varepsilon_{j V}
$$

where the summations are restricted to the nearest neighbors of the vacancy, $n n(V)$, and those of the atom $i$, nn(i). For the sake of simplicity, we assume that an atom $\mathrm{A}$ (or B) in the saddle point position contributes energy $E_{S}{ }^{A}$ (or $\left.E_{S}{ }^{B}\right)$ to the energy of the system.

Further, a vacancy jump is a thermally activated process, and the jump frequency per unit time is given by

$$
\Gamma_{i V}=v \exp \left\{-\frac{\Delta E_{i V}}{k T}\right\}
$$

where the "attempt frequency" $v$ is assumed to be independent of the alloy configuration. The simulation parameters used in this study are listed in Table I. These parameters are determined from experimental data obtained by Soisson et al. [5], and $\varepsilon_{\mathrm{VV}}$ is assumed to be $-0.01 \mathrm{eV}$.

It is natural that the vacancy jump causes an energy decrease toward a more stable state, as shown in Fig. 1-(a). However, if the vacancy is in the minimum state, it has to move from that state with an increase in energy by a certain amount, as shown in Fig. 1-(b).

Here, we define "Monte Carlo time" $\left(=t_{M C}\right)$ in the above vacancy jump model in order to obtain a physical real time scale. $\Gamma_{\mathrm{iv}}$ is the jump frequency per unit time, and the inverse of $\Gamma_{\mathrm{iv}}$ is the time taken at the vacancy jump.

$$
t_{M C}=\frac{1}{\Gamma_{i V}}
$$

However, the vacancy concentration in our simulations is larger than the actual vacancy concentration in pressure vessel steels and $t_{\mathrm{MC}}$ is not the actual time $t$. Then, the actual time $t$ is calculated from $t_{M C}$ using the following linear equation:

$$
t=\frac{C_{V, \text { sim }}}{C_{V, \text { real }}} t_{M C}
$$

Table I Simulation Parameters

\begin{tabular}{|c|c|c|c|c|c|}
\hline$\varepsilon_{\mathrm{FeFe}}$ & $-1.07 \mathrm{eV}$ & $\varepsilon_{\mathrm{CuCu}}$ & $-1.07 \mathrm{eV}$ & $\varepsilon_{\mathrm{FeCu}}$ & $-0.98 \mathrm{eV}$ \\
\hline$\varepsilon_{\mathrm{FeV}}$ & $-0.33 \mathrm{eV}$ & $\varepsilon_{\mathrm{CuV}}$ & $-0.28 \mathrm{eV}$ & $\varepsilon_{\mathrm{VV}}$ & $-0.01 \mathrm{eV}$ \\
\hline $\mathrm{E}_{\mathrm{S}}$ & $-8.9 \mathrm{eV}$ & $v$ & \multicolumn{3}{|c|}{$3.68 \times 10^{15} \mathrm{~s}^{-1}$} \\
\hline
\end{tabular}

where $\mathrm{C}_{\mathrm{v}, \text { sim }}$ and $\mathrm{C}_{\mathrm{v}, \text { real }}$ are the vacancy concentration in the simulation model and the actual vacancy concentration in the pressure vessel steel, respectively.

\subsection{Mechanism of Vacancy Jump}

It can be said that during $\mathrm{Cu}$ precipitation in the actual material, more than two vacancies are involved, because microvoids, namely vacancy clusters, are observed in the $\mathrm{Cu}$ precipitates [5]. Thus, more than two vacancies have to be treated in the present analysis system in order to improve the LMC model. In the conventional LMC model, only one vacancy is introduced, and some numerical technique should be developed in order to introduce more than two vacancies into the model. In this paper, we propose the MVLMC method, in which independent LMC times of the vacancies are unified.

It is clear that if more than two vacancies are introduced into the conventional LMC model, each vacancy has different and independent actual times in the single system. In order to resolve this paradox, we introduce a correction technique in the LMC, which is termed MVLMC. In MVLMC, the actual time calculated for each vacancy is monitored. If the
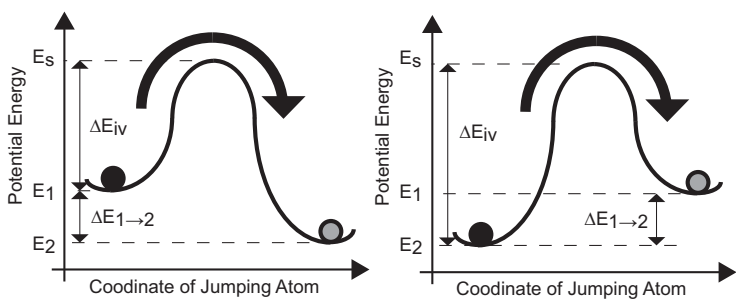

(a) Movement of Vacancy (b) Movement of Vacancy toward a more stable state toward a meta stable state

Fig. 1 Activation Energy of Vacancy Jump Model
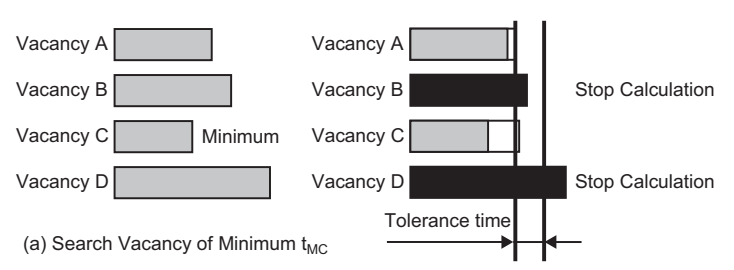

(a) Search Vacancy of Minimum $t_{M C}$

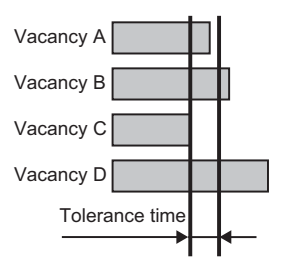

(d) Compare of $\mathrm{t}_{\mathrm{MC}}$ with Tolerance time again

(b) Comparison of $t_{\mathrm{MC}}$ with Tolerance time

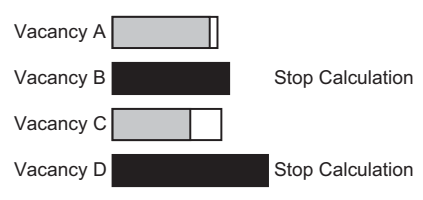

(c) Select of Stop Vacancy

Fig. 2 Concept of Multi Vacancy LMC 
difference between the maximum and minimum actual times exceeds a specified threshold, all LMC calculations except for that of the vacancy with the minimum actual time are paused. Further, if the difference decreases below the threshold, the paused LMC calculations are restarted.

\section{SIMULATION OF Cu PRECIPITATION}

\subsection{Simulation Conditions}

In the present simulation, we firstly arrange Fe atoms in the BCC structure in the form of a matrix and place $\mathrm{Cu}$ atoms in this $\mathrm{Fe}$ matrix. In the initial arrangement of the $\mathrm{Cu}$ atoms, the distributed $\mathrm{Cu}$ atoms are located at a distance greater than the cutoff radius and are not influenced by one another. If we calculate a larger model that contains many atoms on the basis of a 64-bit floating point coordinate system, the computer being used must have a large storage memory. Unfortunately, in our simulation environment, the computer memory is limited. Therefore, we employ a 16-bit integer coordinate system to reduce the memory required by the large model.

We consider that one MVLMC step equals to one vacancy jump, and we calculate up to $3 \times 10^{9}$ steps in the three cases listed in Table II. The other simulation conditions are also listed in this table.

Table II Simulation Conditions A

\begin{tabular}{|c|c|}
\hline Case & 1 \\
\hline Number of Vacancy & 8 \\
\hline Number of Atoms & $20 \times 10^{6}$ \\
\hline Vacancy Concentration & $4.0 \times 10^{-3}[\%]$ \\
\hline Temperature & $600[\mathrm{~K}]$ \\
\hline
\end{tabular}

3.2 Simulation Results and Discussion

The growth and distribution of $\mathrm{Cu}$ clusters in Case 1 are shown in Figs. 3 and 4, respectively. Figures 3-(a) and 4-(a) show that only $\mathrm{Cu}$ clusters smaller than $1 \mathrm{~nm}$ are formed. Figure 3-(a) also shows that the formation of small $\mathrm{Cu}$ clusters is almost saturated in the model. Moreover, Fig. 4-(a) shows that small $\mathrm{Cu}$ clusters form rapidly in the initial stage. Next, Figs. 3-(b) and 4-(b) show that small $\mathrm{Cu}$ clusters disappear gradually and bigger $\mathrm{Cu}$ clusters will appear. Finally, Figs. 3-(c) and 4-(c) show that most of the small $\mathrm{Cu}$ clusters disappear and $\mathrm{Cu}$ clusters larger than $1 \mathrm{~nm}$ are newly formed. The formation mechanism of $\mathrm{Cu}$ clusters will be discussed later.

Next, let us discuss in greater detail the process of $\mathrm{Cu}$ clustering. The "cluster formation fraction" (= (clustered $\mathrm{Cu}$ atoms / all $\mathrm{Cu}$ atoms in model) $\times 100$ ) is calculated; results of a comparison between the cluster formation fraction and total potential energy are shown in Fig. 5. Further, the variation in the mean cluster diameter is also shown in this figure. As shown in the figure, the total potential energy still decreases after the cluster formation fraction becomes almost $100 \%$. From these data, it is considered that $\mathrm{Cu}$ clusters grow such that they not only absorb neighboring atoms but also coalesce with each other. Moreover, from Fig. 6, it is supposed that the mean diameter of clusters is less than $0.8 \mathrm{~nm}$ before the cluster formation fraction becomes almost $100 \%$. After this point, the mean

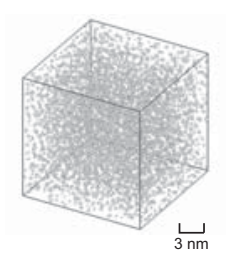

(a) Early Stage

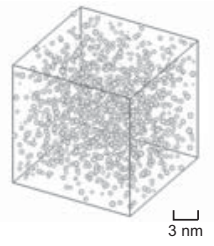

(b) Middle Stage

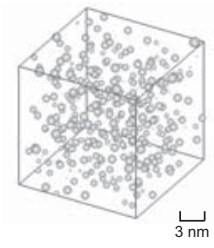

(c) Final Stage
Fig. 3 Growth of Cu Clusters

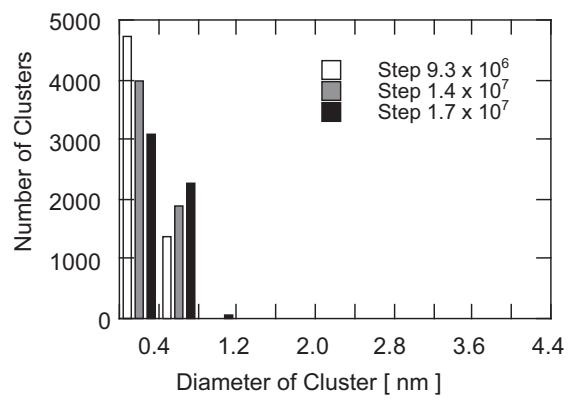

(a) Early Stage

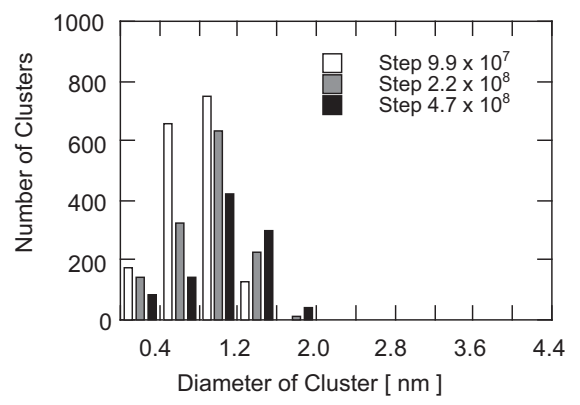

(b) Middle Stage

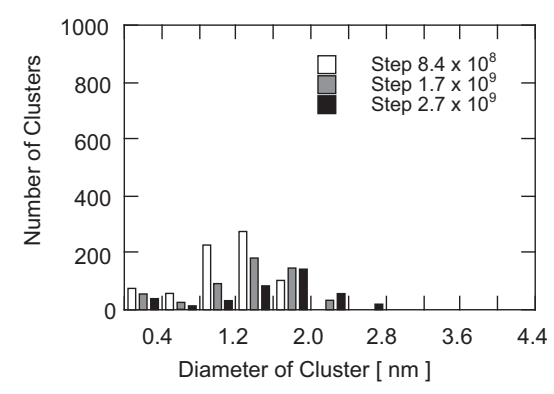

(c) Final Stage

Fig. 4 Distribution of $\mathrm{Cu}$ Clusters

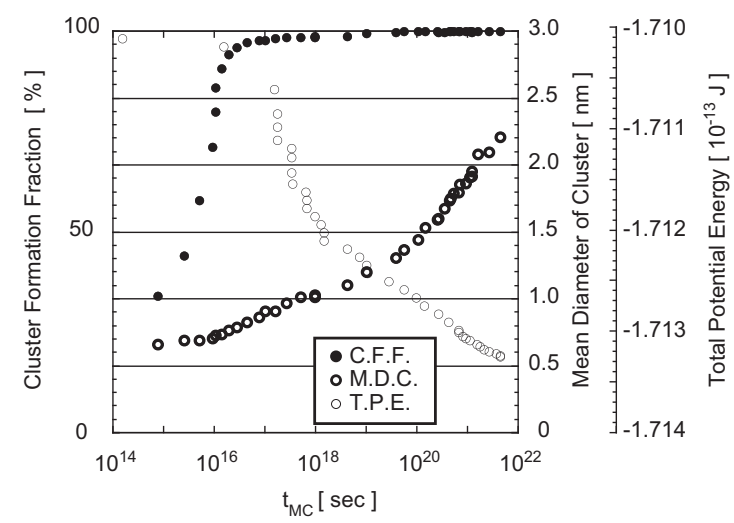

Fig. 5 Variation of Cluster Formation Fraction 
diameter increases rapidly. These observations lead us These discussions lead us to conclude that $\mathrm{Cu}$ clustering occurs in the following two phases.

(I) Formation Phase

In this phase, the cluster formation fraction increases to $100 \%$, and the mean diameter of clusters is less than $0.8 \mathrm{~nm}$.

(II) Coalescence and/or Absorption Phase

In this phase, the cluster formation fraction remains $100 \%$, and the mean diameter of clusters increases rapidly.

\section{SIMULATION OF COALESCENCE AND/OR} ABSORPTION PHASE

4.1 Simulation Conditions

In the present simulation, we firstly arrange Fe atoms in a small BCC structure in the form of a matrix and place two $\mathrm{Cu}$ clusters in this $\mathrm{Fe}$ matrix. We perform calculations in the three cases listed Table III. The other simulation conditions are the same as those listed in Table I.

Table III Simulation Conditions B

\begin{tabular}{|c|c|c|}
\hline Case & $2-1$ & $2-2$ \\
\hline Diameter of Precipitates & 0.8 & 2.0 \\
\cline { 2 - 3 }$[\mathrm{nm}]$ & 1.0 & 2.0 \\
\hline Number of Atoms & \multicolumn{3}{|c|}{16000} \\
\hline Number of Cu Atoms & 74 & 678 \\
\hline Number of Vacancy & \multicolumn{3}{|c|}{4} \\
\hline
\end{tabular}
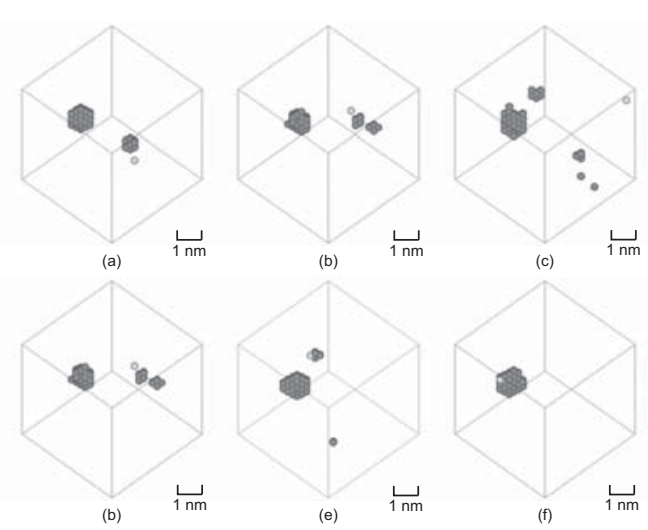

Fig. 6 Movement Clusters in Case 2-1
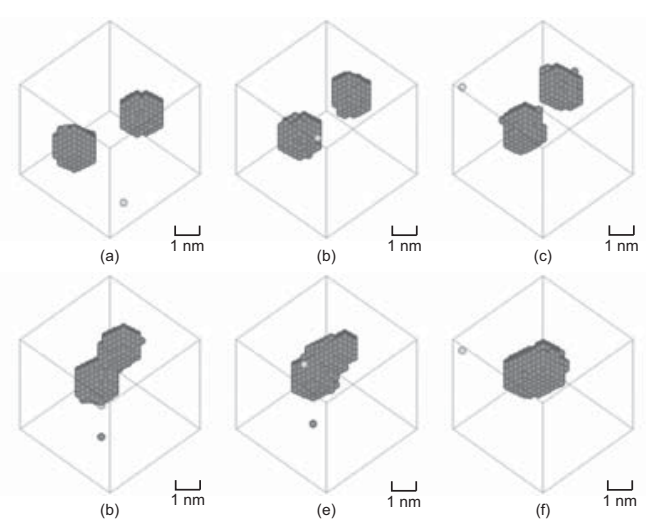

Fig. 7 Movement Clusters in Case 2-2

\subsection{Simulation Result}

The movement of $\mathrm{Cu}$ clusters in Cases 2-1 and 2-2 is shown in Figs. 6 and 7, respectively. A small cluster segregates into an even smaller cluster, which is absorbed as shown in Fig. 8. However, the segregation of the cluster does not occur in the case shown in Fig. 7. Further, the cluster moves after having incorporated a vacancy and then aggregates. Therefore, it is thought that in phase (II), the behavior of clusters changes with a change in their size during the cluster growth process.

\section{CONCLUSIONS}

In order to elucidate the $\mathrm{Cu}$ precipitation process, we simulated a nanoscale $\mathrm{Cu}$ precipitation process on the basis of the vacancy jump model using the developed multi vacancy lattice Monte Carlo method. As a result, we found that process of $\mathrm{Cu}$ clustering consists of the following two phases: phase (I) is the phase of formation of $\mathrm{Cu}$ clusters smaller than $0.8 \mathrm{~nm}$, and phase (II) is the phase of coalescence and/or absorption of small $\mathrm{Cu}$ clusters. Furthermore, in phase (II), the behavior of clusters changes with a change in their size.

\section{ACKNOWLEDGEMENT}

This work was supported by Kougakukiso Co., Ltd.

\section{REFERENCES}

[1] P. J. Othen, M. L. Jenkins and G. D. W. Smith, "High-resolution electron microscopy studies of the structure of $\mathrm{Cu}$ precipitates in $\alpha$-Fe", Phil. Mag. A,Vol. 70, No. 1 (1994), pp.1-24

[2] G.Salge, R.C.Doole, M.Feller-Knipmeier, "High Resolution Electron Microscopy Study of Copper Precipitation in $\mathrm{Fe}-1.5 \mathrm{wt} \% \mathrm{Cu}$ under Electron Irradiation”, J.appl.Phys, Vol.48 (1977), p.1833

[3] H.A.Hardouin Duparc, R.C.Doole, M.L.Jenkins and A.Barbu, "High-Resolution Electron Microscpy Study of Copper Precipitation in $\mathrm{Fe}-1.5 \mathrm{wt} \% \mathrm{Cu}$ under Electron Irradiation”, Phil. Mag. Lett., Vol.71, No. 6 (1995), pp.325-333

[4] Y. Nagai, Z. Tang and M. Hasegawa, "Chemical analysis of precipitates in metallic alloys using coincidence Doppler broadening of positron annihilation radiation", Rad. Phys. Chem., Vol. 58, Issues 5-6 (2000), pp. 737-742

[5] F.Soisson, A.Barbu and G.Martin "Monte Carlo Simulation of Copper Precipitation in Dilute Iron-Copper Alloys During Thermal Ageing and Under Electron Irradiation", Acta Mater, Vol. 44, No. 9, (1996), 3789-3800

[6] S. Schmauder and P. Binkele, "Atomistic computer simulation of the formation of Cu-precipitates in steel", Comput. Mater. Sci., Vol. 24, Issues 1-2 (2002), pp. 42-53

[7] I. Altpeter, G. Dobmann, K. -H. Katerbau, M. Schick, P. Binkele, P. Kizler and S. Schmauder, "Copper precipitates in $15 \mathrm{NiCuMoNb} 5$ (WB 36) steel: material properties and microstructure, atomistic simulation, and micromagnetic NDE techniques", Nucl. Eng. Des., Vol. 206, Issues 2-3 (2001), pp. 337-350

(Received December 9, 2009; Accepted February 23, 2010) 